\title{
SOME COMPLETE INVARIANT METRICS IN GRAUERT TUBES
}

\author{
SU-JEN Kan
}

\begin{abstract}
We first show the existence of the complete invariant Kähler metric $-\log \left(r^{2}-\right.$ $\rho$ ) on the Grauert tube $T^{r} X$ over homogeneous $X$. The existence of a complete KählerEinstein metric of negative Ricci curvature on $T^{r} X$, when $X$ is semi-compact, is also proved. If $X$ is of rank-one symmetric then near the center there exists a Kähler potential depending solely on the Monge-Ampère solution $\sqrt{\rho}$ and the solving of the complete Kähler-Einstein metric is reduced to the solving of an ODE. The restriction to the center of the complete Kähler-Einstein metric is proportional to the original Riemannian metric of $X$ is proved. The holomorphic sectional curvatures of this Kähler-Einstein metric along leaves of the Monge-Ampère foliation near the center is estimated.
\end{abstract}

\section{Introduction.}

It is usually difficult to construct an invariant complete metric. Our goal in this article is to give two complete invariant Kähler metrics in certain kinds of Kähler manifolds, so-called Grauert tubes, and evaluate their curvature behavior.

A Grauert tube $T^{r} X$ is the disk bundle of a Riemannian manifold $X$ of radius $\frac{r}{2}$ equipped with a complex structure which turns every leaf of the Riemannian foliation into a holomorphic curve. The first invariant complete metric discussed is $-\left(\log \left(r^{2}-\rho\right)\right)_{i \bar{j}} d z_{i} d z_{\bar{j}}$ where $4 \rho$ is the length square function; another invariant metric is the complete Kähler-Einstein metric of negative scalar curvature.

For a bounded strictly pseudoconvex domain $D \subset \mathbb{C}^{n}$, Cheng-Yau have proved the existence and uniqueness of a complete Kähler-Einstein metric $g$ of negative scalar curvature by solving a Monge-Ampère type equation. In this article we show the existence of such Kähler-Einstein metrics in $T^{r} X$ provided $X$ is semi-compact.

On studying the existence of complete Ricci-flat metrics on Grauert tubes over compact rank-one symmetric spaces, Stenzel [St] has exploited the two point homogeneity of the center to reduce the Monge-Ampère equation to an ODE.

In Grauert tubes over rank-one symmetric spaces, by using the invariance property and the two-point homogeneity of the center, we construct a Kähler potential of the Kähler-Einstein metric of negative scalar curvature to be a function depending solely in $\rho$. The Monge-Ampère equation defining the Kähler-Einstein metric is an ODE. Thus the solving of the complete Kähler-Einstein metric is reduced to the solving of an explicit ODE in $\rho$.

Received by the editors June 30, 2004. Revised February 2, 2007.

2000 Mathematics Subject Classification. 32C17, 32F29, 32Q20. 
Using some curvature formula derived in $[\mathrm{P}-\mathrm{M}]$ and the asymptotic expansion of $\rho$ near the center, we are able to evaluate the holomorphic sectional sectional curvature of the above Kähler-Einstein metric along leaves of the Monge-Ampère foliation near the center. We test the Grauert tubes $T^{r} R^{n}, T^{r} S^{n}$ and Grauert tubes over the complex projective space, over the quaternionic space and Grauert tubes over the Caley plane. All the above examples have negative holomorphic sectional curvature along the MongeAmpère leaves near the center; we are not sure whether this and the boundary behavior would imply the negativity of the holomorphic sectional curvature along the leaves in the whole Grauert tube.

The organization of this article is the following. In $\S 2$ we prove the existence of a complete invariant Kähler metric and calculate its holomorphic sectional curvature along leaves of the Monge-Ampère foliation. $\S 3$ is on the existence or non-existence of different types of complete Kähler-Einstein metrics. When the center is of rank-one, the potential function of the complete Kähler-Einstein metric of negative scalar curvature is a function of the variable $\rho$ was proved in $\S 4$. In $\S 5$ we show that the defining equation of the above Kähler-Einstein metric can be reduced to an ODE. In $\S 6$, we estimate the holomorphic sectional curvature of this Kähler-Einstein metric along leaves of the Monge-Ampère foliation near the center.

The author would like to thank Yen-Chi Roger Lin for simplifying the proof of Lemma 4.3 .

\section{Grauert tubes and complete invariant Kähler metrics.}

Let $\left(X^{n}, g\right)$ be a connected real-analytic Riemannian manifold. Following [G-S], there exists a neighborhood $U$ of $X$ in the ambient complexified space $\mathbb{C} X$ and a non-negative real-analytic smooth strictly plurisubharmonic function $\rho$ such that $X$ is the zero set of $\rho$ and $(\partial \bar{\partial} \sqrt{\rho})^{n}=0$ on $U-X$. Such a solution $\rho$ is not unique; any positive multiple of $\rho$ still works. To make it unique, some initial conditions are needed. Guillemin-Stenzel have chosen the initial condition as $\left.\left(\frac{\partial^{2} \rho}{\partial z_{i} \partial \bar{z}_{j}}\right)\right|_{X}=2\left(g_{i j}\right)$.

In the equivalent complexification of $[\mathrm{L}-\mathrm{S}]$, the normalization adopted was $\left.\left(\frac{\partial^{2} \rho}{\partial z_{i} \partial \bar{z}_{j}}\right)\right|_{X}=\frac{1}{2}\left(g_{i j}\right)$. In the later case, $\rho$ is exactly the length square of tangent vectors. The normalization we used in $[\mathrm{K} 1]$ and $[\mathrm{K}-\mathrm{M}]$ was $\left.\left(\frac{\partial^{2} \rho}{\partial z_{i} \partial \bar{z}_{j}}\right)\right|_{X}=\left(g_{i j}\right)$. Hence the $\rho$ chosen in this article is actually twice of the $\rho$ in the author's previous articles, i.e., $\left.\left(\frac{\partial^{2} \rho}{\partial z_{i} \partial \bar{z}_{j}}\right)\right|_{X}=$ $2\left(g_{i j}\right)$. The normalization is not important to the rigidity problems. However, it is crucial to the solving of Kähler-Einstein potentials and the completeness of the metrics.

The adapted complex structure in $U$-viewed as an open subset of $T X$-is the unique complex structure which turns every leaf of the Riemannian foliation into a holomorphic curve. The strictly plurisubharmonic function $\rho$ is just 4 times the length-squared function on each tangent vector since in $[\mathrm{L}-\mathrm{S}]$ the $\rho$ is exactly the length-squared function.

A Grauert tube of radius $r$ over center $X$ is defined to be $T^{r} X=\left\{\rho<r^{2}\right\}=$ $\left\{(x, v) \in T X: x \in X,|v|<\frac{r}{2}\right\}$ equipped with the adapted complex structure. Let $r_{\max }(X)$ be the maximal possible radius such that the adapted complex structure could 
be defined. It is clear, for $r<r_{\max }(X)$, that the set

$$
\mathcal{L}:=\left\{(x, v) \in T X: x \in X,|v|_{g}=\frac{r}{2}\right\}
$$

consist of smooth strictly pseudoconvex points. We call $\mathcal{L}$ the strictly pseudoconvex boundary of the Grauert tube $T^{r} X$.

It is clear that $r_{\max }(X)>0$ when $X$ is compact or homogeneous since the adapted complex structure comes from local calculation of the geometry. However, the radius might very well shrink to 0 for non-compact $X$.

Since $\rho$ is strictly plurisubharmonic and $\sqrt{\rho}$ satisfies the homogeneous complex Monge-Ampère equation, $\partial \bar{\partial} \sqrt{\rho}$ has rank $n-1$ on $T^{r} X-X$. Hence ker $\partial \bar{\partial} \sqrt{\rho}$ defines a smooth foliation of $T^{r} X-X$ by Riemannian surfaces, called the Monge-Ampère foliation (c.f. $[\mathrm{B}-\mathrm{K}]$ ). The Grauert tube $T^{r} X$ admits a strictly plurisubharmonic defining function $\rho-r^{2}$. Therefore, the function $h=-\log \left(r^{2}-\rho\right)$ is strictly plurisubharmonic on $T^{r} X$ which induces a Kähler metric $h_{i \bar{j}} d z_{i} d z_{\bar{j}}$ in $T^{r} X$. We would like to see the curvature behavior of this Kähler metric, at least to see the holomorphic sectional curvature along the leaves of the Monge-Ampère foliation. The reference for the following discussion is $[\mathrm{P}-\mathrm{W}]$. First of all, the exhaustion requirement of the Monge-Ampère solution $u$ in the statement of Theorem 3.1 of $[\mathrm{P}-\mathrm{W}]$ is not necessary. They didn't use the exhaustion property for any calculation in $\S 3$ and $\S 4$ of $[\mathrm{P}-\mathrm{W}]$. The exhaustion is only needed in the characterization of a complex manifold possessing certain exhaustion properties stated in Theorem 5.2 of [P-W]. Secondly, there is a typo in the statement of their Theorem 3.2. Let $u$ satisfy the complex homogeneous Monge-Ampère equation away from the $n$-dimensional zero set and $h=f^{-1}(u)$ where $f$ is a real-valued function. Let $Z$ be a vector field such that the leaves of the Monge-Ampère foliation associated to $u$ are the flows of $Z$. The correct form for the holomorphic sectional curvature with respect to the Kähler metric $h_{i \bar{j}} d z_{i} d z_{\bar{j}}$ along the Monge-Ampère leave generated by the vector field $Z$ should be

(2.1) $\mathcal{K}(Z /\|Z\|)=\frac{f^{\prime \prime}(\rho)}{f^{\prime}(\rho)}\left\{2+\frac{\left[f^{\prime}(\rho)\right]^{2}}{\left[f^{\prime \prime}(\rho)\right]^{3}}\left[f^{\prime \prime \prime \prime}(\rho)-2 \frac{\left[f^{\prime \prime \prime}(\rho)\right]^{2}}{f^{\prime \prime}(\rho)}\right]+\left(\frac{f^{\prime}(\rho) f^{\prime \prime \prime}(\rho)}{\left[f^{\prime \prime}(\rho)\right]^{2}}-2\right)^{2}\right\}$.

The typo has occurred due to a misprinting of the term $\left(\frac{f^{\prime}}{f^{\prime \prime}}\right)^{2}$ (should be $\left(\frac{f^{\prime \prime}}{f^{\prime}}\right)^{2}$ instead) and a mix-up of the signature on the calculation of $\mathcal{K}(Z /\|Z\|)$ on p. 370 of $[\mathrm{P}-\mathrm{W}]$. Express the relation as $h=g(u)$ then (2.1) could be simplified as

$$
\mathcal{K}(Z /\|Z\|)=-\frac{g^{\prime \prime}(u) g^{\prime \prime \prime \prime}(u)-\left[g^{\prime \prime \prime}(u)\right]^{2}}{\left[g^{\prime \prime}(u)\right]^{3}} .
$$

The geodesic distance with respect to the $h$-metric from the level set $\{u=a\}$ to the level set $\{u=b\}$ is $\frac{1}{\sqrt{2}} \int_{a}^{b} \sqrt{g^{\prime \prime}(u)} d u$.

Recall that $h$ is defined as $h=-\log \left(r^{2}-\rho\right)$. That is,

$$
u:=\sqrt{\rho}=\left(r^{2}-e^{-h}\right)^{\frac{1}{2}}
$$

satisfies the complex homogeneous Monge-Ampère equation on $T^{r} X-X$.

Let $Z$ be the complex gradient vector field of the Kähler metric $h_{i \bar{j}} d z_{i} d z_{\bar{j}}$. Then, following discussions in $[\mathrm{P}-\mathrm{W}]$, the leaves of the Monge-Ampère foliation associated to $\sqrt{\rho}$ on $T^{r} X$ are the flows of $Z$ and each leaf of the foliation is a totally geodesic submanifold of $T^{r} X$ with respect to the metric $h_{i \bar{j}} d z_{i} d z_{\bar{j}}$. 
Proposition 2.1. Let $X$ be a real-analytic Riemannian manifold such that the Grauert tube $T^{r} X$ exists, $h=-\log \left(r^{2}-\rho\right)$. The holomorphic sectional curvature of the Kähler metric $h_{i \bar{j}} d z_{i} d z_{\bar{j}}$ along the leaves of the Monge-Ampère foliation generated by the vector field $Z$ is

$$
\mathcal{K}(Z /\|Z\|)=-\frac{3 r^{6}+3 r^{4} \rho+9 r^{2} \rho^{2}+\rho^{3}}{\left(r^{2}+\rho\right)^{3}} .
$$

The function $-3 \leq \mathcal{K}(Z /\|Z\|)<-2$ is increasing and it approaches -2 near the strictly pseudoconvex boundary of the Grauert tube.

Proof. From (2.3), $u=f(h)=\left(r^{2}-e^{-h}\right)^{\frac{1}{2}}$. Plugging this $f$ into the formula (2.1), the $\mathcal{K}(Z /\|Z\|)$ is obtained; it is a function depending on the level set $\rho$ alone. The strictly pseudoconvex boundary is the set $\left\{\rho=r^{2}\right\}$ and the holomorphic sectional curvature near the boundary is approaching -1 .

\section{Remark.}

(I). In general, the above metric $-\left(\log \left(r^{2}-\rho\right)\right)_{i \bar{j}}$ is not invariant. However, we may construct an invariant complete metric of similar type if the index of the quotient space $\operatorname{Aut}\left(T^{r} M\right) / A u t_{0}\left(T^{r} M\right)$ is finite. Following the discussion in [K3], we know that $A_{u t}\left(T^{r} M\right)=I_{\operatorname{som}_{0}}(M)$ and we are able to construct an $G$-invariant strictly plurisubharmonic non-negative function $\psi(z)=\sum_{j=1}^{k} \rho\left(g_{j}(z)\right)$ in $T^{r} M$ where $G$ denote $\operatorname{Aut}\left(T^{r} M\right)$ and $\left\{g_{1}=i d, g_{2}, \ldots, g_{k}\right\} \in G$, so that $G / G_{0}=\left\{g_{j} G_{0}: j=1, \ldots, k\right\}$ and $\psi(z) \rightarrow \infty$ as $z$ goes to the strictly pseudoconvex boundary. In this case, the metric $-\left(\log \left(r^{2}-\psi\right)\right)_{i \bar{j}}$ is complete and invariant in $T^{r} M$.

(II). There is a mistake in the proof of Lemma $6.1 \mathrm{in}$ [K3]. A correction is provided in the argument before Proposition 3.5 of [K4]. Nevertheless, Theorem 6.4 [K3] still holds, i.e., $A_{u} t_{0}\left(T^{r} M\right)=\operatorname{Isom}_{0}(M)$ generically.

We'll restrict our attention to homogeneous Riemannian manifolds to guarantee the existence of the Grauert tube structure and the invariance of the above $h$-metric. It was shown in [K3] that over such kind of centers the automorphism group of the Grauert tube $T^{r} X$ is obtained from the differential of the isometry group of $X$ except when $X$ is the real-hyperbolic space and the radius $r=\frac{\pi}{2}$. In other word, if $X$ is homogeneous and $T^{r} X$ is not covered by the ball, then $\operatorname{Aut}\left(T^{r} X\right)=I \operatorname{som}(X)$ for any $r<r_{\max }(X)$.

Throughout this article, we assume that all the centers of Grauert tubes are homogeneous and Grauert tubes are not covered by the ball. We claim that the strictly plurisubharmonic function $\rho$ will induce a complete invariant Kähler metric in $T^{r} X$.

\section{Theorem 2.2.}

Let $(X, g)$ be a homogeneous Riemannian manifold, $\left(h_{i \bar{j}}\right)=-\left(\log \left(r^{2}-\rho\right)\right)_{i \bar{j}}$ be a Kähler metric in $T^{r} X, r<r_{\max }(X)$ and $T^{r} X$ is not covered by the ball. Then

(1) $\left(X, 2 g / r^{2}\right)$ is a totally geodesic submanifold of the Kähler manifold $\left(T^{r} X, h\right)$;

(2) $\left(h_{i \bar{j}}\right)$ is a complete invariant Kähler metric in $T^{r} X$; 
(3) The holomorphic sectional curvature along each leaf of the Monge-Ampère foliation is an increasing function depending on $\rho$, which is bounded by -2 and approaches -2 near the strictly pseudoconvex boundary.

Proof. Since Aut $\left(T^{r} X\right)=\operatorname{Isom}(X)$, the potential function $-\log \left(r^{2}-\rho\right)$ is biholomorphically invariant and hence the induced metric is invariant. Following the lines in $[\mathrm{C}-\mathrm{Y}]$,

$$
h_{i \bar{j}}=\frac{\rho_{i \bar{j}}}{r^{2}-\rho}+\frac{\rho_{i} \rho_{\bar{j}}}{\left(r^{2}-\rho\right)^{2}} .
$$

In local coordinates, $\rho$ has the following asymptotic expansion near $X ; \rho(z)=$ $\sum_{i, j} 4 g_{i j}(x) y_{i} y_{j}+$ h.o.t. The restrictions of $\frac{\partial \rho}{\partial z_{i}}$ and $\frac{\partial \rho}{\partial \bar{z}_{j}}$ to the center $X$ are zero. Therefore, the restriction of the metric $\left(h_{i \bar{j}}\right)$ to $X$ is proportional to $\left(g_{i j}\right)$,

$$
\left.h_{i \bar{j}}\right|_{X}=\frac{\left.\rho_{i \bar{j}}\right|_{X}}{r^{2}}=\frac{2 g_{i j}}{r^{2}} .
$$

The anti-biholomorphism $\tau$ sending $(x, v)$ to $(x,-v)$ is invariant and thus is an isometry of the metric $\left(h_{i \bar{j}}\right)$. Since $X$ is the fixed point set of $\tau$ and the induced metric is proportional to the metric $g,(X, g)$ is a totally geodesic submanifold of the Kähler manifold $\left(T^{r} X, h\right)$.

Let $d_{h}$ denote the distance function on $T^{r} X$ induced from the metric $\left(h_{i \bar{j}}\right)$ and define the metric $d(p, q):=d_{h}(p, q), \forall p, q \in X$. Then, any $l \in \operatorname{Isom}(X)$ is an isometry of $(X, d)$ and any Cauchy sequence in $(X, d)$ converges. Since $\left(h_{i \bar{j}}\right)=-\left(\log \left(r^{2}-\rho\right)\right)_{i \bar{j}}$ is invariant, the proof on the completeness of the Kobayashi metric in Theorem 5.2 of [K3] could be transplanted here. It proves that the metric $\left(h_{i \bar{j}}\right)$ is complete.

The third part is just the restatement of Prop. 2.1.

\section{$\S 3$. Complete Kähler-Einstein metrics on Grauert tubes.}

A Kähler-Einstein metric is a Kähler metric whose Ricci tensor is proportional to the

metric tensor. It is known that every Kähler metric can be locally written as $f_{i \bar{j}}=\frac{\partial^{2} f}{\partial z_{i} \partial \bar{z}_{j}}$ for some potential function $f$. In local coordinates the Ricci tensor associated to this metric is

$$
R_{i \bar{j}}=-\frac{\partial^{2}}{\partial z_{i} \partial \bar{z}_{j}}\left(\log \left(\operatorname{det}\left(f_{k \bar{l}}\right)\right)\right) ;
$$

a Kähler-Einstein metric $\left(f_{i \bar{j}}\right)$ of Ricci curvature $\lambda$ is defined by the following

$$
\frac{\partial^{2}}{\partial z_{i} \partial \bar{z}_{j}}\left(\log \left(\operatorname{det}\left(f_{k \bar{l}}\right)\right)\right)=-\lambda \frac{\partial^{2} f}{\partial z_{i} \partial \bar{z}_{j}} .
$$

There are three cases: $\lambda>0, \lambda=0, \lambda<0$. A classical theorem of Myers stated that there is no complete Kähler-Einstein metric of positive Ricci curvature on a noncompact manifold. We rule out the possibility of having a complete Kähler-Einstein metric of positive Ricci curvature in Grauert tubes. 
A Kähler metric with vanishing Ricci curvature is called Ricci-flat. In [St], Stenzel has shown the existence of complete Ricci-flat metrics on Grauert tubes of infinite radius over compact symmetric spaces of rank-one. Yau has proved in $[\mathrm{Y}]$ a necessary condition, which requires that $M$ does not admit any non-constant bounded holomorphic function, to the existence of a complete Kähler-Einstein metric of non-negative Ricci curvature in a Kähler manifold $M$. Therefore, according to Yau's theorem, there is no non-constant bounded holomorphic function on $T X$ when $X$ is of compact rank-one symmetric. In fact, this holds for any $T X$.

Lemma 3.1. Let $X$ be a real-analytic Riemannian manifold such that the adapted complex structure exists on the whole tangent bundle $T X$. Then there is no non-constant bounded holomorphic function on $T X$.

Proof. By the definition of the adapted complex structure, there exists holomorphic mappings $f: \mathbb{C} \rightarrow T X$ such that the images are Monge-Ampère foliations of $T X$. Suppose there is a bounded holomorphic function $h$ on $T X$. We may assume $h: T X \rightarrow$ $\Delta$ where $\Delta$ is the unit disc in $\mathbb{C}$. Then $h \cdot f$ is a bounded entire function in $\mathbb{C}$ which must be a constant. Hence $h$ is a constant function. A contradiction.

The situation is on the contrary when $r<r_{\max }(X)$.

Lemma 3.2. Let $X$ be a compact Riemannian manifold, $T^{r} X$ be a Grauert tube of radius $r<r_{\max }(X)$. Then there is no complete Ricci-flat Kähler metric on $T^{r} X$.

Proof. Since a Grauert tube over a compact center admits a strictly plurisubharmonic exhaustion function, it is a Stein manifold. $T^{r} X$ is a relatively compact submanifold

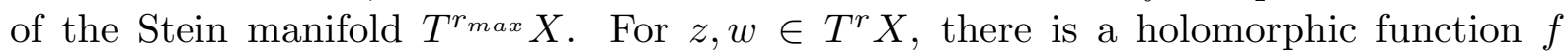
on $T^{r_{\max } X}$ such that $f(z) \neq f(w)$. The restriction function $\left.f\right|_{T^{r} X}$ of $f$ on $T^{r} X$ is a bounded non-constant holomorphic function on $T^{r} X$. Hence there is no complete Ricci-flat metric on $T^{r} X$ for $r<r_{\max }$.

When the centers of Grauert tubes are compact symmetric spaces of rank-one and the radii are $\infty$, Stenzel, in [St], has assured the existence of such complete Ricci-flat metrics. We don't know how the situation goes in $T^{r_{\max }} X, r_{\max }<\infty$ nor in Grauert tubes over non-compact centers. The following two special cases could be seen.

Proposition 3.3. There is no complete Ricci-flat metric in $T^{r} R^{n}, r<\infty$ or in $T^{r} H^{n}$, $r \leq r_{\max }\left(H^{n}\right)$.

Proof. Grauert tubes over the Euclidean space are simply

$$
T^{r} R^{n}=\left\{x+i y \in \mathbb{C}^{n}:|y|^{2}=\sum_{j=1}^{n} y_{j}^{2}<r^{2}\right\} .
$$

It is clear that

$$
T^{r} R^{n} \subset\left\{z_{1} \in \mathbb{C}: y_{1}^{2}<r^{2}\right\} \times \cdots \times\left\{z_{n} \in \mathbb{C}: y_{n}^{2}<r^{2}\right\} .
$$


By the Riemann Mapping Theorem, $T^{r} R^{n}$ is a domain in $\Delta^{n} \subset \mathbb{C}^{n}$, where $\Delta$ represents the unit disc in $\mathbb{C}$. Define a function $f(z)=\sum_{j=1}^{n} z_{j}$ in $\Delta^{n}$. The restriction of $f$ to $T^{r} R^{n}$ is a bounded non-constant holomorphic function in $T^{r} R^{n}$.

Recall from its construction on p. 357 of $[\mathrm{K}-\mathrm{M}]$, Grauert tubes over $H^{n}$ could be expressed as

$$
D_{s}=\left\{w \in \mathbb{C}^{n}:\left|w_{1}\right|^{2}+\cdots+\left|w_{n}\right|^{2}-1<s\left|w_{1}^{2}+\cdots+w_{n}^{2}-1\right|\right\}
$$

where $s \in(-1,1]$. The maximal Grauert tube is $D_{1}$ and $1-w_{1}^{2}-\cdots-w_{n}^{2}$ cannot be 0 or a negative real number. We specify the branch for the square root function to be the one with argument in $[0, \pi)$, then

$$
f(w):=\frac{1}{1-i \sqrt{1-w_{1}^{2}-\cdots-w_{n}^{2}}}
$$

is a bounded non-constant holomorphic function in $T^{r} H^{n}, r \leq r_{\max }\left(H^{n}\right)$.

In [C-Y], the authors have introduced the concept of bounded geometry, a generalization of strictly pseudoconvexity, to facilitate the solvability of a Monge-Ampère type equation which is necessary for the existence of Kähler-Einstein metrics. We would like to see the existence of such kinds of Kähler-Einstein metrics in Grauert tubes of various types.

Definition. A real-analytic Riemannian manifold $(X, g)$ is semi-compact if there exists a compact subset $K \subset M$ such that $I \operatorname{som}(M) \cdot K=M$.

It is clear that all compact Riemannian manifolds, homogeneous Riemannian manifolds and products of them are all included in this family. Furthermore, $r_{\max }(X)>0$ if $X$ is semi-compact.

Theorem 3.4. Let $X^{n}$ be a semi-compact Riemannian manifold, $T^{r} X$ be a Grauert tube of radius $r<r_{\max }(X)$. Then there is a unique complete real-analytic KählerEinstein metric of Ricci curvature $-(n+1)$ on $T^{r} X$. The holomorphic sectional curvature approaches to -1 near the strictly pseudoconvex boundary points.

Proof. The metric $\left(h_{i \bar{j}}\right)=-\left(\log \left(r^{2}-\rho\right)\right)_{i \bar{j}}$ is a complete $\operatorname{Isom}(X)$-invariant Kähler metric in $T^{r} X$. Since $X$ is semi-compact, there exists a compact subset $K \subset X$ such that $\operatorname{Isom}(X) \cdot K=X$. The far ends of $X$ and tangent vectors over them would be well under control. The other part to be taken care of is the set

$$
\left\{(x, v): v \in T_{x} X,|v|=\frac{r}{2}\right\}
$$

consisting of smooth strictly pseudoconvex boundary points. Since $h$ is $\operatorname{Isom}(X)$ invariant and every point in $X$ could be shifted to a point in the compact set $K$ through an isometry, it is clear that such a complete Kähler manifold $\left(T^{r} X, h_{i \bar{j}}\right)$ is of bounded geometry as defined by Cheng and Yau. Furthermore, the function $-\left(\log \left(r^{2}-\rho\right)\right)$ is strictly 
plurisubharmonic in the whole manifold $T^{r} X$. Therefore, following the discussion of [C$\mathrm{Y}]$ on p.519, there exists a unique function $u$ such that $\left(-\left(\log \left(r^{2}-\rho\right)\right)_{i \bar{j}}+u_{i \bar{j}}\right)>0$ and

$$
\operatorname{det}\left(-\left(\log \left(r^{2}-\rho\right)\right)_{i \bar{j}}+u_{i \bar{j}}\right)=e^{(n+1) u} e^{-(n+1) \log \left(r^{2}-\rho\right)} .
$$

The metric $\left(-\left(\log \left(r^{2}-\rho\right)\right)_{i \bar{j}}+u_{i \bar{j}}\right)$ is the unique complete Kähler-Einstein metric of Ricci curvature $-(n+1)$ in $T^{r} X$. Since $\rho$ is real-analytic, the real-analyticity of the Kähler-Einstein metric follows from Theorem 4.4 of $[\mathrm{C}-\mathrm{Y}]$.

Remark. When $X$ is compact, the above radius is allowed to be $r=r_{\max }(X)$ following the argument of $[\mathrm{C}-\mathrm{Y}]$ on exhausting a bounded pseudoconvex domain by bounded strictly pseudoconvex domains.

The uniqueness of a Kähler-Einstein metric implies that it is invariant with respect to any biholomorphic map. It is also clear that this metric is invariant under any antibiholomorphism of the Grauert tube. That is, any anti-biholomorphism is an isometry of this metric. Therefore, the fixed point set of an anti-biholomorphism equipped with the reduced metric is a totally geodesic submanifold. Take the natural anti-holomorphic involution of the Grauert tube $T^{r} X$, then $\left(X, h_{X}\right)$ is a totally geodesic submanifold of the Kähler-Einstein manifold $\left(T^{r} X, h\right)$ where $h_{X}:=\left.h\right|_{X}$.

Lemma 3.5. Suppose there is a Kähler potential depending real-analytically on the function $\rho$ alone in a Grauert tube $T^{r} X$. Then the restriction of the Kähler metric to the $X$ is a metric proportional to the original Riemannian metric $\left(g_{i \bar{j}}\right)$ of $X$.

Proof. It is clear by the real-analyticity of the potential function, the fact that $\left.\left(\frac{\partial^{2} \rho}{\partial z_{i} \partial \bar{z}_{j}}\right)\right|_{X}=2\left(g_{i j}\right)$ and that on the center $\rho=0, \frac{\partial \rho}{\partial z_{j}}=0$ and $\frac{\partial \rho}{\partial \bar{z}_{j}}=0, \forall j=1, \cdots, n$.

Besides the ball case, it is almost impossible to get any explicit solution from (3.2). Stenzel is the first one who has successfully simplified the equation (3.2) on certain Grauert tubes to an ODE for the case $\lambda=0$ by examining the special properties occurred from the complex homogeneous Monge-Ampère equation defining the Grauert tubes. Later on, R. Aguilar discussed the possibility of writing the Kähler potential in terms of the radius of the Grauert tubes. We'll show that if $X$ is a compact symmetric space of rank-one, is $R^{n}$, or is $H^{n}$, then there is a potential function in $T^{r} X$ for the complete Kähler-Einstein metricof Ricci $-(n+1)$ depending on $\rho$ alone. Furthermore, the defining equation (3.2) is reduced to an ODE.

\section{$\S 4$. Potential functions depending on the radius.}

Consider the complete Kähler-Einstein metric $k$ of Ricci $-(n+1)$ on the unit ball $B=\left\{\left(z_{1}, \cdots, z_{n}\right) \in \mathbb{C}^{n}:\left|z_{1}\right|^{2}+\cdots+\left|z_{n}\right|^{2}<1\right\}$. A standard potential function for $k$ is $f=-\log \left(1-\left|z_{1}\right|^{2}-\cdots-\left|z_{n}\right|^{2}\right)$. The unit ball is a Grauert tube over the hyperbolic space of radius $\frac{\pi}{2}$ which is half the maximal radius. In terms of projective coordinates, the ball is biholomorphic to

$$
\begin{aligned}
M_{0}=\left\{w \in \mathbb{C}^{n+1}:\right. & w_{1}^{2}+\cdots+w_{n}^{2}-w_{n+1}^{2}=-1, \\
& \left.\left|w_{1}\right|^{2}+\cdots+\left|w_{n}\right|^{2}-\left|w_{n+1}\right|^{2}<0\right\} .
\end{aligned}
$$


The Monge-Ampère solution $\sqrt{\rho}$ in the model $M_{0}$ was given at (7.12) of [K1](with slightly different scaling), $\eta:=\left|w_{1}\right|^{2}+\cdots+\left|w_{n}\right|^{2}-\left|w_{n+1}\right|^{2}=-\cos \sqrt{\rho}$. The biholomorphism from $M_{0}$ to $B^{n}$ is given as $z_{j}=w_{j} / w_{n+1}, j=1, \cdots, n$. The above $\eta$ is then $\eta=\frac{\left|z_{1}\right|^{2}+\cdots+\left|z_{n}\right|^{2}-1}{\left|z_{1}^{2}+\cdots+z_{n}^{2}-1\right|}$. The potential function

$$
\begin{aligned}
f & =-\log \left(1-\left|z_{1}\right|^{2}-\cdots-\left|z_{n}\right|^{2}\right) \\
& =-\log (-\eta)\left|z_{1}^{2}+\cdots+z_{n}^{2}-1\right| \\
& =-\log (-\eta)-\log \left|z_{1}^{2}+\cdots+z_{n}^{2}-1\right| .
\end{aligned}
$$

Since $\partial \bar{\partial} \log \left|z_{1}^{2}+\cdots+z_{n}^{2}-1\right|=0$, the function $f$ and $-\log (-\eta)=-\log (\cos \sqrt{\rho})$, a function of $\rho$ alone, are in the same Kähler class. Therefore, they define the same Kähler metric.

Giving a compact Lie group $G$ and a closed subgroup $K$ of $G$. In [Sz], Szőke has shown the biholomorphic equivalence of $G_{\mathbb{C}} / K_{\mathbb{C}}$ and the tangent bundle $T(G / K)$ equipped with the adapted complex structure associated to the bi-invariant metric on $G / K$. Putting a further restriction on the Lie groups, $G$ is semi-simple and $K$ is closed and connected then it was proved by Stenzel $\left[\mathrm{St}\right.$ ] that there exists a $G_{\mathbb{C}}$-invariant non-vanishing holomorphic $(n, 0)$ section $\omega$ in $G_{\mathbb{C}} / K_{\mathbb{C}}=T(G / K)$, the Grauert tube over $G / K$ of infinite radius. The whole class of compact symmetric spaces of rank-one has fulfilled the above condition. That is, there exists an $G$-invariant nowhere vanishing holomorphic $(n, 0)$ form $\mathcal{V}$ on the whole tangent bundle $T G$ when $G$ is a compact symmetric space of rankone. Since $G$ is the isometry group and a Grauert tube is invariant under the action of the isometry group of the center manifold, this $\mathcal{V}$ is an $G$-invariant nowhere vanishing holomorphic $(n, 0)$ form when restricted to any finite Grauert tubes $T^{r} G, r<\infty$.

It is not clear at all whether this kind of holomorphic $(n, 0)$ forms exist in Grauert tubes over generic real-analytic Riemannian manifolds. Nevertheless, it is not hard to see that such kind of $(n, 0)$ forms do exist in Grauert tubes of small radii over any homogeneous Riemannian manifold. Let $(X, g)$ be a homogeneous Riemannian manifold and let $\mathcal{V}$ denote the Riemannian volume form, the unique $n$-form which has the value 1 at any orthonormal basis, of $(X, g)$. It is clear that $\mathcal{V}$ is isometrically invariant and is real-analytic. Let $\mathcal{V}^{\mathbb{C}}$ be the complexification of $\mathcal{V}$. Since the geometric properties of a homogeneous Riemannian manifold is controlled within a compact subset, there exists a small $\epsilon>0$ such that $\mathcal{V}^{\mathbb{C}}$ is a well-defined nowhere vanishing $\operatorname{Isom}(X)$-invariant holomorphic $(n, 0)$ form in $T^{\epsilon} X$. By the rigidity of the Grauert tubes over homogeneous Riemannian manifolds, $\mathcal{V}^{\mathbb{C}}$ is also biholomorphically invariant. We summarize the above discussion as the following lemma.

Lemma 4.2. Let $X$ be a homogeneous Riemannian manifold. Then there exists a nowhere vanishing invariant holomorphic $(n, 0)$ form on $T^{\epsilon} X$ for $\epsilon<<1$.

It is interesting to see how far this $\epsilon$ could go. It is clear that $\epsilon$ could be chosen to be any positive number when $X$ is a compact symmetric space of rank-one. It is also clear that this holds for the Euclidean space $R^{n}$ since the invariant Riemannian volume form is $\mathcal{V}=d x_{1} \wedge d x_{2} \cdots \wedge d x_{n}$. Its complexification $\mathcal{V}^{\mathbb{C}}=d z_{1} \wedge d z_{2} \cdots \wedge d z_{n}$ is an invariant holomorphic $(n, 0)$ form in the Grauert tube

$$
T^{r} R^{n}=\left\{z=x+i y \in \mathbb{C}^{n}:|y|<r\right\} .
$$


The desired $(n, 0)$ form also exists in $T^{r} H^{n}$. Let

$$
H^{n}=\left\{x \in R^{n+1}: x_{1}^{2}+x_{2}^{2}+\cdots+x_{n}^{2}-x_{n+1}^{2}=-1, x_{n+1}>0\right\} .
$$

The quadratic form $\sum_{j=1}^{n} d x_{j}^{2}-d x_{n+1}^{2}$ has induced on $H^{n}$ a postive definite symmetric 2-tensor $\mathcal{L}_{g}$. For $r \in(0, \pi]$,

$$
\begin{aligned}
T^{r} H^{n}=\left\{z \in \mathbb{C}^{n+1}: z_{1}^{2}+\cdots+z_{n}^{2}-z_{n+1}^{2}=-1\right. \\
\left.\left|z_{1}\right|^{2}+\cdots+\left|z_{n}\right|^{2}-\left|z_{n+1}\right|^{2}<-\cos r\right\} .
\end{aligned}
$$

The strategy is to find the Riemannian volume form in $H^{n}$ and then complexify it to a holomorphic $(n, 0)$ form in $T^{r} H^{n}$. Consider the isometric map

$$
\psi:\left(R^{n}, g\right) \rightarrow\left(H^{n}, \mathcal{L}_{g}\right), \quad \psi\left(x_{1}, \cdots, x_{n}\right)=\left(x_{1}, \cdots, x_{n},\left(1+\sum_{j=1}^{n} x_{j}^{2}\right)^{\frac{1}{2}}\right)
$$

where $g=\left(g_{i j}\right)$ is the pulled back of the metric $\mathcal{L}_{g}$;

$$
g_{i j}=\delta_{i j}-\frac{x_{i} x_{j}}{1+\sum_{k=1}^{n} x_{k}^{2}} .
$$

Lemma 4.3. $\operatorname{det}\left(g_{i j}\right)=\frac{1}{1+\sum_{k=1}^{n} x_{k}^{2}}$.

Proof. Let $A:=\left(\frac{x_{i} x_{j}}{1+\sum_{k=1}^{n} x_{k}^{2}}\right)$ be an $n \times n$ matrix and let $\left\{\lambda_{j}\right\}_{j=1}^{n}$ be eigenvalues of $A$. Then the eigenvalues of $\left(g_{i j}\right)$ are $\left\{1-\lambda_{j}\right\}_{j=1}^{n}$ since $\left(g_{i j}\right)=I-A$ and both matrices share the same eigenvectors. Taking out the common factors of each row and then subtracting the $j$-rows by the first row. It is clear that the matrix $A$ has rank one, i.e., $A$ has $n-1$ zero eigenvalues. Since the trace is the sum of all eigenvalues, we conclude that the non-zero eigenvalue of $A$ is $\frac{\sum_{k=1}^{n} x_{k}^{2}}{1+\sum_{k=1}^{n} x_{k}^{2}}$. Therefore the matrix $\left(g_{i j}\right)$ has $n-1$ eigenvalues 1 and one eigenvalue

$$
1-\frac{\sum_{k=1}^{n} x_{k}^{2}}{1+\sum_{k=1}^{n} x_{k}^{2}}=\frac{1}{1+\sum_{k=1}^{n} x_{k}^{2}} .
$$

Therefore, $\operatorname{det}\left(g_{i j}\right)=\frac{1}{1+\sum_{k=1}^{n} x_{k}^{2}}$.

Let $\mathcal{V}$ denote the Riemannian volume form in the model $\left(R^{n}, g\right)$ and $\hat{\mathcal{V}}$ the corresponding volume form in $\left(H^{n}, \mathcal{L}_{g}\right)$,

$$
\begin{aligned}
& \mathcal{V}=\sqrt{\left(\operatorname{det}\left(g_{i j}\right)\right)} d x_{1} \wedge d x_{2} \cdots \wedge d x_{n}=\frac{1}{\sqrt{1+\sum_{k=1}^{n} x_{k}^{2}}} d x_{1} \wedge d x_{2} \cdots \wedge d x_{n} \\
& \hat{\mathcal{V}}=\frac{1}{\sqrt{1+\sum_{k=1}^{n} x_{k}^{2}}} d x_{1} \wedge d x_{2} \cdots \wedge d x_{n}=\frac{1}{x_{n+1}} d x_{1} \wedge d x_{2} \cdots \wedge d x_{n} .
\end{aligned}
$$

Since $\mathcal{V}$ is isometrically invariant and $\psi$ is an isometry, the volume form $\hat{\mathcal{V}}$ is a nowhere vanishing isometrically invariant $(n, 0)$ form in $\left(H^{n}, \mathcal{L}_{g}\right)$. That is, $\hat{\mathcal{V}}$ is an $S O_{+}(n, 1)$ invariant nowhere vanishing real-analytic $n$-form in $\left(H^{n}, \mathcal{L}_{g}\right)$. The symbol $S O_{+}(n, 1)$ denotes the subgroup of $S O(n, 1)$ which keeps $H^{n}$ invariant, which is the isometry group of $\left(H^{n}, \mathcal{L}_{g}\right)$. Let

$$
\hat{\mathcal{V}}^{\complement}=\frac{1}{z_{n+1}} d z_{1} \wedge d z_{2} \cdots \wedge d z_{n}
$$

be the complexification of $\hat{\mathcal{V}}$. 
Lemma 4.4. $\left.\hat{\mathcal{V}}^{\mathbb{C}}\right|_{T^{r} H^{n}}$ is a nowhere vanishing invariant holomorphic $(n, 0)$ form in the Grauert tube $T^{r} H^{n}$ for all $r \in(0, \pi]$.

Proof. Let $A \in S O_{+}(n, 1), z=x+i y \in T^{r} H^{n}$, the action of $A$ on $z$ is defined as $A z=A x+i A y$. By the characteristic property of $S O(n, 1)$, it could be checked that $T^{r} H^{n}$ is invariant under the action of $S O_{+}(n, 1)$.

Since $\operatorname{Aut}\left(T^{r} H^{n}\right)=\operatorname{Isom}\left(H^{n}\right),\left.\hat{\mathcal{V}}^{\mathbb{C}}\right|_{T^{r} H^{n}}$ is naturally an invariant holomorphic $(n, 0)$ form. The existence of $\hat{\mathcal{V}}^{\mathrm{C}}$ is equivalent to the nonvanishing of $z_{n+1}$. From the definition of $T^{r} H^{n}$ in (4.1), we see that

$$
x_{1}^{2}+\cdots+x_{n}^{2}-x_{n+1}^{2}<\frac{-1-\cos r}{2} \leq 0 .
$$

Hence $x_{n+1}=\Im z_{n+1} \neq 0$ throughout the whole tube $T^{r} H^{n}$.

Two crucial points in [St] to prove the existence of a complete Ricci-flat metric are the existence of a non-vanishing globally defined holomorphic $(n, 0)$ form and the isotropy of the center. They are essential in simplifying (3.2) to an ODE.

Theorem 4.5. Let $X$ be a symmetric space of rank-one, $k=\left(k_{i \bar{j}}\right)$ be the KählerEinstein metric of Ricci $-(n+1)$ in the Grauert tube $T^{r} X$. Then there exists an $\epsilon>0$ and a potential function $h$ for the metric $\left(k_{i \bar{j}}\right)$ such that the restriction of $h$ to $T^{\epsilon} X$ depends real-analytically on $\rho$. The $\epsilon$ could be chosen to be equal to $r$ when $X$ is compact, $X$ is the Euclidean plane $R^{n}$ or the real-hyperbolic space $H^{n}$.

Proof. Let $f$ be a Kähler potential of the Kähler-Einstein metric $k$, by definition

$$
\operatorname{Ric}(k)=-\partial \bar{\partial} \log \operatorname{det}\left(k_{i \bar{j}}\right)=-(n+1) \partial \bar{\partial} f .
$$

Let $\epsilon$ be as in Lemma 4.2. We take it to be $r$ in the case $r$ is less $\epsilon$. By the rigidity of Grauert tubes over homogeneous spaces, $\operatorname{Aut}\left(T^{\epsilon} X\right)=\operatorname{Isom}(X)=\operatorname{Aut}\left(T^{r} X\right)$.

Since $k$ is an invariant metric, $\partial \bar{\partial} f$ is invariant under the action of $\operatorname{Aut}\left(T^{r} X\right)=$ $\operatorname{Isom}(X):=I$. Thus $\left.\partial \bar{\partial} f\right|_{T^{\epsilon} X}$ is also $I$-invariant. Let $\mathcal{V}$ be the holomorphic $I$-invariant $(n, 0)$ form in $T^{\epsilon} X$ constructed in Lemma 4.2, then $\mathcal{V} \wedge \overline{\mathcal{V}}$ is a nowhere vanishing $I$ invariant $(n, n)$ form in $T^{\epsilon} X$. Thus there is an $I$-invariant function $F$ on $T^{\epsilon} X$ such that

$$
\left(\left.\partial \bar{\partial} f\right|_{T^{\epsilon} X}\right)^{n}=F \mathcal{V} \wedge \overline{\mathcal{V}}
$$

The transitivity and the rank-one condition of $I$ imply that $I$ map each level set $\{\rho=c\}$ onto the level set itself. Therefore, $F$ is $I$-invariant and $F$ is a function of $\rho$. In local coordinates $\left(z_{1}, \cdots, z_{n}\right), \mathcal{V}=\zeta(z) d z_{1} \wedge \cdots \wedge d z_{n}$ for a holomorphic function $\zeta$ and (4.7) is read as

$$
\operatorname{det}\left(\frac{\partial^{2} f}{\partial z_{k} \partial \bar{z}_{l}}\right)=F(\rho)|\zeta|^{2}
$$


The equation (4.6) holds as well when the defining function $f$ is restricted to $T^{\epsilon} X$. Comparing (4.6) and (4.8), on $T^{\epsilon} X$,

$$
\frac{\partial^{2} f}{\partial z_{i} \partial \bar{z}_{j}}=\frac{1}{n+1} \frac{\partial^{2}}{\partial z_{i} \partial \bar{z}_{j}} \log F(\rho)|\zeta|^{2}=\frac{1}{n+1} \frac{\partial^{2}}{\partial z_{i} \partial \bar{z}_{j}} \log F(\rho) .
$$

Hence $h:=\frac{1}{n+1} \log F(\rho)$ is a potential of the Kähler-Einstein metric $k$ depending solely on $\rho$ when restricted to $T^{\epsilon} X$.

The rest of the theorem is clear since such an invariant nowhere vanishing holomorphic $(n, 0)$ form exists in $T^{r} X$ for any $r \leq r_{\max }(X)$ for the listed three cases.

\section{Reduction to an ordinary differential equation.}

Given a Riemannian manifold $(X, g)$, the Riemannian density function $\mathcal{S}$ of $X$ is defined as follows. In polar coordinates with $p$ as the origin the volume element of the geodesic ball centered at $p$ of radius $r$ is given by $r^{n-1} \mathcal{S}_{p} d v$ where $d v$ is the measure on $S^{n-1}$. A Riemannian manifold $(X, g)$ is harmonic if the density function $\mathcal{S}$ solely depends on the geodesic distance. Standard examples are symmetric spaces of rank-one.

In [A], Aguilar has viewed the real-analytic Riemannian manifold $X$ as the diagonal of the product manifold $X \times X$ and then extended the real-analytic objects through the analytic complexification to the complex manifold $X_{\mathbb{C}} \times X_{\mathbb{C}}$. He showed if there is a Kähler-Einstein potential depending solely on the Monge-Ampère solution $\sqrt{\rho}$ in the Grauert tube $T^{r} X$, then the Kähler potentials of such kind of Kähler-Einstein metrics satisfy an ODE near the center. By the uniqueness of analytic continuation, if there exists a potential function for a Kähler-Einstein metric in a Grauert tube which depends solely on the Monge-Ampère solution $\sqrt{\rho}$ then the potential function satisfies an ODE in the whole tube.

Since a rank-one symmetric space is harmonic and homogeneous, there exists a Kähler potential, solely depending on the length square function $\rho$, for the complete KählerEinstein metric of negative Ricci in $T^{r} X$. Such a potential function is defined by the following ODE

$$
\hat{h}^{\prime \prime}(u)\left(\hat{h}^{\prime}(u)\right)^{(n-1)} \exp (-(n+1) \hat{h}(u))=C_{r} u^{n-1} \hat{\mathcal{S}}(u),
$$

$\hat{h}(r)=\infty, C_{r}$ is a non-zero constant depending on $T^{r} X$ and $\hat{h}$.

Let $h=\hat{h}+\frac{\log C_{r}}{(n+1)}$ which is also a Kähler potential of the complete Kähler-Einstein metric of scalar curvature $-(n+1)$. $h$ satisfies the following ODE as described in Prop. 5.1 of $[\mathrm{A}]$,

$$
h^{\prime \prime}(u)\left(h^{\prime}(u)\right)^{(n-1)} \exp (-(n+1) h(u))=u^{n-1} \hat{\mathcal{S}}(u) .
$$

The derivatives are taken with respect to the Monge-Ampère solution $u=\sqrt{\rho} ; \hat{\mathcal{S}}(u):=$ $\mathcal{S}\left(-u^{2}\right)$ where $\mathcal{S}$ is the density function of the respective center manifold. Hence, $\hat{\mathcal{S}}$ is a real-analytic function of $\rho$. By Theorem 4.5, $h$ is a real-analytic function of $\rho$. Take derivatives with respect to the variable $\rho$ and rewrite (5.1) as

$$
\left(h^{\prime}(\rho)\right)^{n}+2 \rho\left(h^{\prime}(\rho)\right)^{n-1} h^{\prime \prime}(\rho)=e^{(n+1) h(\rho)} \hat{\mathcal{S}}(\sqrt{\rho}) .
$$


Proposition 5.1. Let $X^{n}$ be a symmetric space of rank-one and let $k$ be the complete Kähler-Einstein metric of Ricci curvature - $(n+1)$ in the Grauert tube $T^{\epsilon} X=\left\{\rho<\epsilon^{2}\right\}$ as described in Lemma 4.2. Then the Kähler-Einstein metric $k$ has a unique realanalytic Kähler potential $h$ such that $h$ is a solution of the equation (5.2) with the boundary condition $h\left(\epsilon^{2}\right)=\infty$ and $\frac{d^{2} h}{d \rho^{2}}(0)>0$. The $\epsilon$ could be chosen to be any number less than $r_{\max }(X)$ when $X$ is compact, $X$ is $R^{n}$ or $H^{n}$.

Proof. Since the Kähler-Einstein metric comes from the perturbation of the defining function $-\log \left(\epsilon^{2}-\rho\right)$, the boundary condition $h\left(\epsilon^{2}\right)=\infty$ follows. Since $h$ is a realanalytic function of $\rho$, the condition $h^{\prime \prime}(0)>0$ follows from Lemma 3.5. Denote $u=\sqrt{\rho}$. Suppose $f(u)$ and $g(u)$ are potential functions of a complete Kähler-Einstein metric of Ricci $-(n+1)$ in the Grauert tube $T^{\epsilon} X$. Let $F(u)=f(u)-g(u)$, by the uniqueness of the complete Kähler-Einstein metric of negative scalar curvature,

$$
\partial \bar{\partial} F(u)=0 .
$$

Away from the set $\{u=0\}$, using the fact that $(\partial \bar{\partial} u)^{n}=0$,

$$
(\partial \bar{\partial} F(u))^{n}=n F^{\prime \prime}(u)\left(F^{\prime}(u)\right)^{n-1} \partial u \wedge \bar{\partial} u \wedge(\partial \bar{\partial} u)^{n-1} .
$$

The non-vanishing of $\partial u \wedge \bar{\partial} u \wedge(\partial \bar{\partial} u)^{n-1}$ implies $F^{\prime \prime}(u)\left(F^{\prime}(u)\right)^{n-1}=0$ which shows that $F(u)=c u+d$ for some constants $c$ and $d$. The condition (5.3) will force $c$ to be zero since $\partial \bar{\partial} u$ is non-vanishing. However if $d \neq 0, g(u)$ and $f(u)=g(u)+d$ can't both be solutions of (5.1) unless the density function is identically zero which is a contradiction. Therefore, $d=0$ and the uniqueness follows.

Remark. For $n=1, T^{r} X$, by Riemannian mapping theorem, is biholomorphic to the unit disc in $\mathbb{C}$ and the equation (5.1) descends to $h^{\prime \prime} e^{-2 h}=1$ which has a simple solution $h(\rho)=-\log \left(r^{2}-\rho\right)$ on $T^{r} X$. There is a simple solution $h=-\log \cos (\sqrt{\rho})$ for $(5.1)$ at $r=\frac{\pi}{2}$, which coincides with the example in $\S 4$ since $T^{\frac{\pi}{2}} H^{n}$ is biholomorphic to the unit ball.

\section{Curvature behavior near the center.}

As a Grauert tube is foliated by Monge-Ampère foliations, it is interesting to see how curvatures behave along the Monge-Ampère leaves and near the center. In [K2], the author has proved that the rigidity of the Grauert tube holds if the Kähler-Einstein metric is negatively curved, i.e., if all sectional curvatures of the Kähler-Einstein metric are negative everywhere, the Grauert tube has a unique center. It is a naive guessing that this negativity occurs for all Grauert tubes. We will show the sectional curvature is proportional to the sectional curvature of the original Riemannian metric, hence it could be of any signature.

Lemma 6.1. Let $(X, g)$ be a rank-one symmetric space, $k$ be the complete KählerEinstein metric of Ricci- $-(n+1)$ in $T^{\epsilon} X$. Then sectional curvatures of $k$ on $X$ are proportional to sectional curvatures of $g$ on $X$.

Proof. Since $\left(X,\left.k\right|_{X}\right)$ is totally geodesic in $\left(T^{r} X, k\right)$, the curvature behavior of the metric $k$ on $X$ is the same as the metric $\left.k\right|_{X}$ on $X$. By Prop. 5.1 and Lemma 3.5, $\left.k\right|_{X}$ is proportional to the Riemannian metric $g$. The result follows. 
Lemma 6.2. Let $h(u)$ be a potential function of a Kähler metric in a Grauert tube $T^{r} X$ with an asymptotic expansion $h(u)=a+b u^{2}+c u^{4}+O(6), b \neq 0$, near the center $X$. Then, the holomorphic sectional curvature $\mathcal{K}$ along the Monge-Ampère leaves near the center of the tube is $\frac{-6 c}{b^{2}}$.

Proof. A direct calculation from (2.2) shows that near the center the holomorphic sectional curvature along the leaves is

$$
\frac{\left(h^{\prime \prime \prime}\right)^{2}-h^{\prime \prime} h^{\prime \prime \prime \prime}}{\left(h^{\prime \prime}\right)^{3}}=-6 \frac{c\left(b-6 c u^{2}\right)}{\left(b+6 c u^{2}\right)^{3}}
$$

which goes to $\frac{-6 c}{b^{2}}$ as $u \rightarrow 0$.

We are able to estimate the holomorphic sectional curvature for a couple of cases.

Proposition 6.3. Let $X$ be the Euclidean space or the unit sphere, $k$ be the complete Kähler-Einstein metric on the Grauert tube $T^{r} X$ of Ricci curvature $-(n+1)$. Then, near the center, $k$ has negative holomorphic sectional curvature along the Monge-Ampère leaves.

Proof. Let $h(u)$ denote the unique real-analytic potential function of the Kähler-Einstein metric $k$ described in Proposition 5.1. $h(u)$ has to satisfy the ordinary differential equation (5.1) with suitable $\hat{\mathcal{S}}(u)$. As $h$ is a real-analytic function of $\rho$, we may write the asymptotic expansion of $h$ near the center as

$$
h(u)=a+b u^{2}+c u^{4}+O(6) .
$$

By lemmas 3.5 and $6.1, b>0$.

Comparing the asymptotic expansions of the both sides of (5.1), we have

$$
\hat{\mathcal{S}}(u)=1, c=\frac{b^{2}(n+1)}{2 n+4}
$$

for the flat case and

$$
\hat{\mathcal{S}}(u)=\left(\frac{\sinh u}{u}\right)^{(n-1)}, c=\frac{(n-1) b+6(n+1) b^{2}}{6(4+2 n)}
$$

for the sphere case. Both $c$ are positive, by Lemma 6.2 the holomorphic sectional curvatures are negative.

When $X=H^{n}$ the corresponding density function and $c$ are

$$
\hat{\mathcal{S}}(u)=\left(\frac{\sin u}{u}\right)^{n-1}, c=\frac{-(n-1) b+6(n+1) b^{2}}{6(4+2 n)} .
$$

This $c$ is positive only if $b>\frac{n-1}{6(n+1)}$. On the other hand $b=\frac{1}{2} \exp \frac{n+1}{n} a$, the size of $b$ is controlled by $a$. If $a$ is very negative then $b$ will not satisfy the inequality $b>\frac{n-1}{6(n+1)}$. 
It seems the boundary condition for $h$ at $u=\epsilon$ doesn't give much information about what happens at $u=0$.

The situation is quite simple for other compact rank-one symmetric spaces. For the complex projective space

$$
\hat{\mathcal{S}}(u)=2^{n-1} u^{n-1} \cosh \frac{u}{2}\left(\sinh \frac{u}{2}\right)^{n-1}, c=\frac{(n+2) b+24(n+1) b^{2}}{24(4+2 n)} ;
$$

for the quaternionic projective space

$$
\hat{\mathcal{S}}(u)=2^{n-1} u^{n-1}\left(\cosh \frac{u}{2}\right)^{3}\left(\sinh \frac{u}{2}\right)^{n-1}, c=\frac{(n+8) b+24(n+1) b^{2}}{24(4+2 n)} ;
$$

for the Caley plane

$$
\hat{\mathcal{S}}(u)=2^{n-1} u^{n-1}\left(\cosh \frac{u}{2}\right)^{7}\left(\sinh \frac{u}{2}\right)^{n-1}, c=\frac{(n+20) b+24(n+1) b^{2}}{24(4+2 n)} .
$$

Since $b$ is positive, $c>0$ for all the above three cases. The holomorphic sectional curvatures of the complete Kähler-Einstein metric of Ricci - $(n+1)$ of the Grauert tube $T^{r} X$ along the Monge-Ampère leaves near the center is negative when $X$ is the complex projective space, the quaternionic projective space or the Caley plane.

\section{References}

[A] R. Aguilar, Pseudo-Riemannian metrics, Kähler-Einstein metrics on Grauert tubes and harmonic Riemannian manifolds, Q. J. Math. 51, no. 1 (2000), 1-17.

[B-K] E. Bedford and M. Kalka, Foliations and complex Monge-Ampère equation, Comm. Pure Appl. Math. 30 (1977), 543-571.

[C-Y] S.-Y. Cheng and S.-T. Yau, On the existence of a complete Kähler metric on non-compact complex manifolds and the regularity of Fefferman's equation, Comm. Pure. Appl. Math. 33 (1980), 507-544.

[G-S] V. Guillemin \& M. Stenzel, Grauert tubes and the homogeneous Monge-Ampère equation, J.Diff. Geom. 34 (1991), 561-570.

[K1] S.-J. Kan, The asymptotic expansion of a CR invariant and Grauert tubes, Math. Ann. 304 (1996), 63-92.

[K2] - On the rigidity of non-positively curved Grauert tubes, Math. Z. 229 (1998), 349-363.

[K3] On rigidity of Grauert tubes over homogeneous Riemannian manifolds, J. reine angew. Math. 577 (2004), 213-233.

[K4] Complete hyperbolic Stein manifolds with prescribed automorphism groups, Comment. Math. Helv. 82 No. 2 (2007), 371-383.

[K-M] S.-J. Kan and D. Ma, On rigidity of Grauert tubes over Riemannian manifolds of constant curvature, Math. Z. 239 (2002), 353-363.

[L-S] L. Lempert \& R. Szöke, Global solutions of the homogeneous complex Monge-Ampère equations and complex structures on the tangent bundle of Riemannian manifolds, Math.Ann. 290 (1991), 689-712. 
[P-W] G. Patrizio \& P.-M. Wong, Stein manifolds with compact symmetric centers, Math.Ann. 289 (1991), 355-382.

[St] M. Stenzel, Ricci-flat metrics in the complexification of a compact rank one symmetric space, Manuscripta Math. 80 (1993), 151-163.

[Sz] R. Szőke, Adapted complex structures and Riemannian homogeneous spaces, Ann. Polon. Math. 70 (1998), 215-220.

[Y] S.-T. Yau, A general Schwarz lemma for Kähler manifolds, Amer. J. Math. 100, No. 1 (1978), 197-203.

Institute of Mathematics, Academia Sinica, Taipei, Taiwan

E-mail address: kan@math.sinica.edu.tw 